\title{
Impacto de las Abejas (Apis mellifera L.) Como Agentes Polinizadores en el Rendimiento del Cultivo de Zucchini (Cucurbita pepo L.) en el Canton Riobamba, Provincia de Chimborazo
}

\author{
Herrera Villalobos M. J., \\ Ing. Agronóma de la Escuela Superior Politécnica de Chimborazo, Ecuador \\ Lindao Córdova V. A., \\ Doctor en Ciencias Ambientales de la Universidad Nacional San Marcos \\ Lima Perú, Profesor a Tiempo Completo de la Escuela Superior Politécnica \\ de Chimborazo, Ecuador \\ Espinosa Espinosa A. E., \\ Master en Ciencias: Profesor a Tiempo Completo de la Escuela Superior \\ Politécnica de Chimborazo, Ecuador \\ Carrera Guanoluisa E. R., \\ Yanéz Villacís J., \\ Ing. Agronómo: Técnico Docente, \\ de la Escuela Superior Politécnica de Chimborazo, Ecuador
}

Doi:10.19044/esj.2019.v15n24p207 URL:http://dx.doi.org/10.19044/esj.2019.v15n24p207

\section{Resumen}

Se evaluó el impacto de las abejas (Apis mellifera L.) como agentes polinizadores en el rendimiento del cultivo de zucchini (Cucurbita pepo L.) en el cantòn Riobamba, provincia de Chimborazo. Para llevar a cabo esta evaluación se utilizó un diseño de bloques completos a azar (DBCA), con tres tratamientos y tres repeticiones. Los tratamientos evaluados fueron: El cultivo de zucchini dentro del micro túnel cubierto con malla entomológica con presencia de abejas, el cultivo de zucchini dentro del micro túnel cubierto con malla antiáfidossin presencia de abejas y el cultivo de zucchini a campo abierto. Los parámetros evaluados fueron número de días transcurridos después de la aparición de los frutos, porcentaje de flores fecundadas y no fecundadas, número de frutos por planta, tamaño de frutos, diámetro de frutos, peso del fruto y rendimiento en $\mathrm{kg} / \mathrm{ha}$ por tratamiento. Los mejores resultados alcanzados en la mayoría de los parámetros evaluados como el porcentaje de floración, número de frutos por planta, tamaño de fruto en $(\mathrm{cm})$, diámetro de fruto en $(\mathrm{cm})$, peso del fruto en gramos, rendimiento en $\mathrm{kg} / \mathrm{ha}$ por tratamiento y análisis económico, se obtuvieron con la presencia de las abejas (Apis 
mellifera L.) como agentes polinizadores en el túnel con mallas durante la etapa de floración del cultivo de zucchini, con un rendimiento en promedio de $14.9 \mathrm{tn} / \mathrm{ha}$.

Palabras clave: Zucchini, Polinización, Fertilizados

\title{
Impact of bees (Apis mellifera L.) as Pollinating Agents in the Performance of The Zucchini Crop (Cucurbita pepo L.) in the Canton Riobamba, Province of Chimborazo
}

\section{Herrera Villalobos M. J.,}

Ing. Agronóma de la Escuela Superior Politécnica de Chimborazo, Ecuador

Lindao Córdova $V$. A.,

Doctor en Ciencias Ambientales de la Universidad Nacional San Marcos Lima Perú, Profesor a Tiempo Completo de la Escuela Superior Politécnica de Chimborazo, Ecuador

Espinosa Espinosa A. E.,

Master en Ciencias: Profesor a Tiempo Completo de la Escuela Superior

Politécnica de Chimborazo, Ecuador

Carrera Guanoluisa E. R., Yanéz Villacís J.,

Ing. Agronómo: Técnico Docente, de la Escuela Superior Politécnica de Chimborazo, Ecuador

\begin{abstract}
The impact of bees (Apis mellifera L.) as pollinators in the yield of the zucchini (Cucurbita pepo L.) crop in the Riobamba canton, Chimborazo province, was evaluated using a Randomized Complete Block Design (RCBD). This was done using three treatments and three repetitions. The treatments evaluated were: the zucchini cultivation inside the micro tunnel covered with entomological mesh with the presence of bees, the zucchini cultivation inside the micro tunnel covered with anti aphids mesh without the presence of bees, and the open field zucchini cultivation. The evaluated parameters were number of days to the appearance of the fruits, percentage of fertilized and unfertilized flowers, number of fruits per plant, size of fruits, diameter of fruits, weight of fruit, and yield in $\mathrm{kg} /$ ha per treatment. The best
\end{abstract}


results achieved in most parameters that were evaluated such as the percentage of flowering, number of fruits per plant, size of fruit in $\mathrm{cm}$, diameter of fruit in $\mathrm{cm}$, weight of the fruit in grams, yield in $\mathrm{kg} / \mathrm{ha}$ by treatment, and economic analysis were obtained with the presence of bees (Apis mellifera L.) as pollinating agents in the tunnel. This is in addition to meshes during the flowering stage of the zucchini crop, with an average yield of $14.9 \mathrm{tn} / \mathrm{ha}$.

Keywords: Zucchini, Pollination, Fertilized

\section{Introducción}

Las plantas de zucchini (Cucurbita pepo L.) presentan una particularidad relevante respecto a su expresión sexual, ya que son mayoritariamente monoicas; es decir, las flores masculinas y femeninas están separadas en una misma planta, apareciendo generalmente las masculinas anticipadamente a las femeninas. Las flores masculinas aparecen generalmente en una proporción mayor a las femeninas, a la vez que, de las femeninas, sólo llegan a ser cosechadas como frutos del 20 al $50 \%$ de ellas. En parte, este resultado es consecuencia de las variables que interactúan en la eficiencia de la polinización. Los granos de polen son pesados, pegajosos y no están adaptados al transporte por el viento, por ello, la polinización es necesariamente entomófila. Las abejas (Apis mellifera L.) son los principales insectos que intervienen en la polinización de cultivos comerciales (Zaccari, 2004).

La polinización por las abejas no sólo incrementa la producción de los cultivos, sino que también mejora la calidad, asegurando el máximo tamaño y rendimiento de los frutos si se llevan suficientes colmenas y las condiciones de clima no afectan el pecoreo (Reyes \& Cano, 2005). Por ejemplo, en el cultivo de zucchini con la polinización de las abejas se pueden lograr incrementos de los rendimientos del 20 al $30 \%$ (Moacho, 2011).

Dado que más del $75 \%$ de los cultivos del mundo dependen de polinizadores, la disminución de sus poblaciones ha generado gran alerta sobre las consecuencias de esta pérdida. Si la interacción planta-polinizador se rompe, podríamos dejar de tener acceso a cientos de frutas, verduras y legumbres que hacen parte de nuestra dieta actual y se afectarían los servicios ambientales derivados de la función ecológica de la polinización. El servicio de polinización biótica es prestado por diversos grupos de animales incluyendo mamíferos, aves e insectos. Los insectos, con una riqueza entre 2,5 y 3,7 millones de especies, son los polinizadores más importantes tanto en ecosistemas naturales como en agro ecosistemas (Hamilton et al., 2010).

Las abejas son los insectos que por excelencia participan en esta labor, por lo que poseen una gran importancia económica y ecológica en los agroecosistemas; de hecho, una gran parte de los alimentos que hoy en día se 
consumen y comercializan masivamente, dependen directa o indirectamente de la polinización realizada por abejas (FAO, 2014).

El uso de $A$. mellifera L. en el mundo está destinado principalmente a la producción de miel, siendo la renta de colmenas para polinización de cultivos otra forma de uso ampliamente establecida en varios países del mundo. En los países latinoamericanos, la explotación de Apis mellifera L. se centra principalmente en la obtención y comercialización de miel y polen, dándole menor importancia a la polinización de cultivos (Garibaldi et al., 2013).

Existen cultivos cuya producción sería prácticamente nula en ausencia de polinizadores, como el cacao, la nuez y la vainilla. Las cucurbitáceas (zapallos, sandías, melones, etc.) también constituyen un ejemplo de alta dependencia de polinizadores, particularmente de abejas, que actúan de manera efectiva en sus enormes flores (Kevan, 2003).

En pocas palabras, la biodiversidad es necesaria en la agricultura para cultivar alimentos. Se calcula que uno de cada tres bocados de nuestros alimentos depende de la polinización. Sin biodiversidad de insectos, los agricultores tendrían que llevar a cabo la polinización manualmente, algo que costaría miles de millones de dólares cada año. Resalta la función que estos desempeñan en los ecosistemas naturales y los agro-ecosistemas, enfatizando la necesidad de tomar medidas para su conservación (Kevan, 2003).

\section{Materiales y métodos}

El área de estudio está ubicada en invernaderos tipo túnel en la parroquia Licán (Latitud de 9816945 UTM, Longitud: 758141 UTM y Altitud de 2834 msnm), Cantón Riobamba, Provincia de Chimborazo.

Se utilizó un Diseño de Bloques Completos al Azar (DBCA), con tres tratamientos T1 (cultivo de zucchini dentro del micro túnel cubierto con malla entomológica con presencia de abejas para la polinización), T2 (cultivo de zucchini edentro del micro túnel cubierta con malla antiáfidos en el cual no se permitió el ingreso de ningún insecto sin presencia de abejas), y T3 (cultivo de zucchini a campo abierto) con tres repeticiones, dando un total de 9 unidades experimentales. Se realizó el análisis de varianza, se determinó el coeficiente de variación para cada una de las variables y se expresó en porcentaje y se ejecutó el análisis económico utilizando la relación beneficio/costo de los tratamientos. Se efectuó igualmente la prueba de TUKEY al 5\% cuando existieron diferencias significativas entre los tratamientos. Se investigó la acción de las abejas en el rendimiento del cultivo de zucchini y se expresó el rendimiento en $\mathrm{kg} / \mathrm{ha}$.

En el área total del ensayo se construyeron dos invernaderos tipo mini túnel de 24,70 m de largo x $3 \mathrm{~m}$ de ancho y $1.70 \mathrm{~m}$ de altura, en cuyo interior se cultivo el zucchini. En el tratamiento T1 el túnel estuvo cubierto con malla 
entomológica para impedir la salida de abejas y se introdujo una colmena de (Apis mellifera L) con 400 individuos; el tratamiento T2 se cubrió con una malla antiáfidos para impedir el ingreso de insectos de ningúna especie y el tratamiento T3 el zucchini se cultivó a campo abierto. Se determinó la parcela neta en cada uno de los tres tratamientos y se sortearon 10 plantas por tratamiento para su seguimiento y evaluación.

Se contabilizó y se registró por seis semanas el número de días transcurridos desde la polinización a la aparición de los frutos de las 10 plantas marcadas al azar de cada parcela neta.

A los 68 días, cuando las plantas presentaron el $10 \%$ de floración, se inició el conteo de flores fecundadas hasta alcanzar su máxima floración a los 104 días después de la siembra y se expresó en porcentaje, aplicando la siguiente fórmula de Martínez (2009):

$\%$ de flores fecundadas $=$ (Número de flores fecundadas $/$ Número de flores totales) $x 100$

El porcentaje de flores no fecundadas se registró visualmente a partir de los 68 días, cuando las plantas presentaron el $10 \%$ de floración. Posteriormente se inició el conteo de flores fecundadas hasta alcanzar su máxima floración a los 104 días después de la siembra de las 10 plantas marcadas al azar de cada parcela neta y se expresó en porcentaje aplicando la siguiente fórmula:

$\%$ de flores no fecundadas $=($ Número de flores no fecundadas $/$ Número de flores totales) $x 100$

El número de frutos de las 10 plantas marcadas al azar de cada parcela neta se contabilizó a la cosecha a partir de los 78 días después de la siembra, cuando los frutos alcanzaron su madurez fisiológica, durante seis semanas.

El tamaño del fruto de las 10 plantas marcadas al azar de cada parcela neta se determinó en cada cosecha durante seis semanas con la ayuda de una cinta métrica y se expresó en cm.

El diámetro de fruto de las 10 plantas marcadas al azar de cada parcela neta se determinó en cada cosecha durante seis semanas con la ayuda de un calibrador y se expresó en cm.

El peso de los frutos de las 10 plantas marcadas al azar de cada parcela neta se determinó en cada cosecha durante seis semanas con la ayuda de una balanza y se expresó en gramos.

El rendimiento de las 10 plantas marcadas al azar se determinó en cada cosecha durante seis semanas, y se expresó en $\mathrm{kg} /$ parcela neta y luego se proyectó a $\mathrm{kg} / \mathrm{ha}$. 


\section{Resultados y discusión}

\subsection{Número de días transcurridos a la aparición de los frutos desde la} polinización

El análisis de varianza para el número de días transcurridos después de la aparición de los frutos desde la polinización (Tabla 1) presentó diferencias altamente significativas para los tratamientos con un p-valor $<0,0001$ y con un coeficiente de variación de $0.60 \%$.

Tabla 1. Análisis de varianza para el número de días transcurridos después de la aparición de los frutos desde la polinización

\begin{tabular}{|c|c|c|c|c|c|}
\hline F.V. & SC & GL & CM & F & P-valor \\
\hline Repeticiones & 0,003 & 2 & 0,0017 & 6,43 & 0,0563 \\
Tratamientos & 1,170 & 2 & 0,590 & 2247,87 & $<0,0001$ \\
Error & 0,001 & 4 & 0,00026 & & \\
Total & 1,180 & 8 & & & \\
\hline C.V. 0,60\% & \multicolumn{5}{|l}{} \\
\hline
\end{tabular}

En la prueba de Tukey al 5\% para el número de días transcurridos después de la aparición de los fruto desde la polinización (Tabla 2) se presentan tres rangos: en el rango "A" se encuentra el tratamiento T1 (túnel con malla entomológica con abejas), con una media de 2,26 días, en el rango "C" se encuentra el tratamiento T2 (túnel con malla antiáfidossin la presencia de insectos) con 3.15 días.

Tabla 2. Prueba de Tukey al 5\% para el número de días transcurridos después de la aparición de los frutos desde la polinización

\begin{tabular}{|l|l|l|}
\hline Tratamiento & Medias & Rango \\
\hline T1 (Túnel con malla entomológica) & 2,26 & A \\
T3 (Campo abierto) & 2,66 & B \\
T2 (Túnel con malla antiáfidos sin la presencia de insectos & 3,15 & C \\
\hline
\end{tabular}

Fuente: Herrera (2018)

El menor número de días transcurridos después de la aparición de los frutos desde la polinización, se presentó en el tratamiento T1 (túnel con malla entomológica con abejas) con una media de 2.26 días; esto puede deberse a la acción de las abejas que pueden recorrer grandes distancia en segundos. Además, estos insectos recolectores de néctar para su alimentación, pueden transportar los granos de polen de la flor masculina a la femenina debido a que se adhieren en sus patas y abdomen, contribuyendo de esta manera con la polinización y como consecuencia con la formación de frutos de calidad. La temperatura dentro del túnel, que fue de $25^{\circ} \mathrm{C}$, permitió que las flores masculinas y femeninas se mantuvieran abiertas al mismo tiempo, lo que pudo contribuir a la aparición de los frutos en menor tiempo. Esto concuerda con Noriega (2003) quien indica que el cultivo de zucchini requiere en la etapa de floración temperaturas entre 20 a $25^{\circ} \mathrm{C}$, y con Rylski y Alón (1990), quienes manifiestan que, para una polinización acertada, tanto las flores masculinas 
como las femeninas deben estar abiertas durante el mismo día. En días fríos las flores femeninas abren antes que las masculinas, retrasando el cuaje.

El mayor número de días transcurridos después de la aparición de los frutos desde la polinización (Cuadro 2) corresponde al tratamiento T2 (túnel protegido con malla antiáfidos sin presencia de abejas) con una media de 3.15 dias. Esto puede deberse posiblemente a la no presencia de insectos polinizadores que ayuden a transportar los granos de polen de la flor masculina a la femenina, a la temperatura y humedad relativamente elevadas dentro del túnel que alcanzaron $\operatorname{los} 33^{\circ} \mathrm{C}$ y $60 \%$ respectivamente, los pocos frutos fecundados que aparecieron posiblemente pueden haberse formado por el viento o la acción mecánica producida al momento de la evaluación, coincidiendo con Nepi y Pacini (1993), quienes manifiestan que la transferencia mecánica del polen es esencial para el cuaje del fruto.

\subsection{Porcentaje de flores fecundadas}

En el análisis de varianza para el porcentaje de flores fecundadas (Tabla 3) se encuentran diferencias altamente significativas para tratamientos con un p-valor < 0,0001; su coeficiente de variación alcanzo 0,76\%.

Tabla 3. Análisis de varianza para el porcentaje de flores fecundadas

\begin{tabular}{|c|c|c|c|c|c|}
\hline F.V. & SC & GL & CM & $\mathbf{F}$ & P-valor \\
\hline Repeticiones & 0,46 & 2 & 0,23 & 1,02 & 0,4383 \\
\hline Tratamientos & 2824,70 & 2 & 1412,35 & 6259,49 & $<0,0001$ \\
\hline Error & 0,90 & 4 & 0,23 & & \\
\hline Total & 2826,06 & 8 & & & \\
\hline C.V. $0,76 \%$ & & & & & \\
\hline
\end{tabular}

En la prueba de Tukey al 5\% para el porcentaje de flores fecundadas en el Tabla 4se presentan tres rangos: en el rango "A" se encuentra el tratamiento T1 (túnel con malla entomológica), con una media de 84,27\%, en el rango "C" se encuentra el tratamiento T2 (túnel con malla antiáfidos), con $40,89 \%$ de flores fecundadas.

Tabla 4. Prueba de Tukey al 5\% para el porcentaje de flores fecundadas

\begin{tabular}{|c|c|c|}
\hline Tratamiento & Medias & Rango \\
\hline (T1) Túnel con malla entomológica & 84,27 & $\mathrm{~A}$ \\
(T3) Campo abierto & 61,39 & $\mathrm{~B}$ \\
(T2) Túnel con malla antiáfidos & 40,89 & $\mathrm{C}$ \\
\hline
\end{tabular}

Fuente: Herrera (2018)

El alto porcentaje de flores fecundadas en el T1 (túnel con malla entomológica con abejas) con una media de $84,27 \%$, puede deberse a la presencia de abejas, las cuales al recolectar el néctar y el polen actúan comoagentes polinizadores transportando los granos de polen de la flor masculina a la femenina, propiciando así la fecundación de las flores 
femeninas y por ende el cuajado de los frutos del zucchini. Este resultado coincide con Reyes y Cano (2005) quienes mencionan que las abejas melíferas son los más eficientes polinizadores, pues visitan muchas flores de la misma especie en sucesión, se mueven frecuentemente de una flor a otra, llenan sus cuerpos peludos de polen y lo llevan a otras flores, efectuando así una transferencia muy efectiva.

En el tratamiento T2 (túnel protegido con malla antiáfidos sin presencia de abejas), el porcentaje de fecundación de las flores fue bajo con una media de $40.9 \%$, esto debido a que no hubo presencia de las abejas ni de otros insectos polinizadores. Zaccari (2004) manifiesta que las abejas constituyen los agentes más eficientes en la polinización de las cucurbitáceas. El uso de insecticidas, herbicidas y las prácticas de cultivo han reducido o eliminado las poblaciones silvestres de insectos hasta el punto de hacerlos insuficientes para la polinización de plantaciones comerciales. Así, los productores de cultivos hortícolas y frutales prácticamente dependen de la abeja para cumplir con los requerimientos de polinización.

\subsection{Porcentaje de flores no fecundadas}

En el análisis de varianza para el porcentaje de flores no fecundadas en la Tabla 5 se encuentran diferencias altamente significativas para tratamientos con un p-valor < 0,0001; su coeficiente de variación alcanzó un $2.28 \%$.

Tabla 5. Análisis de varianza para el porcentaje de flores no fecundadas

\begin{tabular}{|c|c|c|c|c|c|}
\hline F.V. & SC & GL & CM & F & P-valor \\
\hline Repeticiones & 2,05 & 2 & 1,03 & 1,52 & 0,3233 \\
Tratamientos & 3157,20 & 2 & 1578,60 & 2333,25 & $<0,0001$ \\
Error & 2,71 & 4 & 0,68 & & \\
Total & 3161,96 & 8 & & & \\
\hline C.V. $0,76 \%$ & \multicolumn{5}{|l}{} \\
\hline \multicolumn{7}{|c|}{$\mathrm{p}<0,05^{*} ; \mathrm{p}<0,01^{* *} ; \mathrm{p}>0,05 \mathrm{~ns}$} \\
\end{tabular}

En la prueba de Tukey al 5\% para el porcentaje de flores no fecundadas (Tabla 6) se presentan tres rangos: en el rango "A" se encuentra el tratamiento T1 (túnel con malla entomológica) con una media de 13,23\% y en el rango "C" el T2 (túnel con malla antiáfidos) con una media $59,11 \%$ de flores no fecundadas.

Tabla 6. Prueba de Tukey al 5\% para el porcentaje de flores no fecundadas

\begin{tabular}{|c|c|c|}
\hline Tratamiento & Medias & Rango \\
\hline (T1) Túnel con malla entomológica & 13,23 & $\mathrm{~A}$ \\
(T3) Campo abierto & 35,85 & $\mathrm{~B}$ \\
(T2) Túnel con malla antiáfidos & 59,11 & $\mathrm{C}$ \\
\hline
\end{tabular}

Fuente: Herrera (2018)

El menor porcentaje de flores no fecundadas en el cultivo de zucchini (Cuadro 6), lo presentó el tratamiento T1( túnel con malla entomológica). 
Esto pudo deberse a la presencia de abejas dado que son los más eficientes polinizadores de las flores en cucurbitáceas, lo que coincide con Arizmendi (2009), quien manifiesta que las tres cuartas partes de los cultivos de los que se alimenta el hombre, dependen de la polinización para producir sus frutos. Se calcula que sin los polinizadores no se podría tener uno de cada tres bocados de comida que se consumen. Entre los cultivos importantes que requieren polinizadores están el frejol, el tomate, las calabacitas, los frutales, etc. Igualmente, cultivos como la alfalfa, de los que depende la producción de carne, necesitan polinizadores para producir semillas. El $80 \%$ de cultivos depende de un polinizador para su producción, y en el $12 \%$ de ellos es esencial la polinización; es decir, de no existir los polinizadores no se obtendrían los frutos o las semillas.

El tratamiento T2 (túnel con malla antiáfidos), presentó mayor cantidad de flores no fecundadas con una media de 59.1, debido a que no existe la presencia de las abejas, la cual reduce las posibilidades de que un número elevado de flores queden fecundadas.

\subsection{Número de frutos por planta}

En el análisis de varianza para el número de frutos por planta (Tabla 7), se encuentran diferencias altamente significativas para los tratamientos con un p-valor < 0,0001; su coeficiente de variación alcanzó un 3,04\%.

Tabla 7. Análisis de varianza para el número de frutos por planta

\begin{tabular}{|c|c|c|c|c|c|}
\hline F.V. & SC & GL & CM & F & P-valor \\
\hline Repeticiones & 0,10 & 2 & 0,05 & 2,46 & 0,2013 \\
Tratamientos & 24,92 & 2 & 12,46 & 640,69 & $<0,0001$ \\
Error & 0,08 & 4 & 0,02 & & \\
Total & 25,09 & 8 & & & \\
\hline C.V. 3,04\% & $\mathrm{p}<0,05 * ; \mathrm{p}<0,01 * * ; \mathrm{p}>0,05 \mathrm{~ns}$
\end{tabular}

En la prueba de Tukey al 5\% para el número de frutos por planta (Tabla 8 ) se presentan tres rangos, en el rango " $\mathrm{A}$ " se encuentra el tratamiento T1(túnel con malla entomológica) con una media de 6,70 y en el rango " $\mathrm{C}$ " el T2 (túnel con malla antiáfidos) con una media de 2,63 frutos por planta.

Tabla 8. Prueba de Tukey al 5\% para el número de frutos por planta

\begin{tabular}{|c|c|c|}
\hline Tratamiento & Medias & Rango \\
\hline (T1) Túnel con malla entomológica & 6,70 & $\mathrm{~A}$ \\
(T3) Campo abierto & 4,43 & $\mathrm{~B}$ \\
(T2) Túnel con malla antiáfidos & 2,63 & $\mathrm{C}$ \\
\hline
\end{tabular}

El tratamiento T1 (túnel con malla entomológica) con presencia de abejas (Tabla 8), es el que presentó mayor número de frutos cosechados con una media de 6.7 por planta. Esto puede deberse a una polinización eficiente 
de las abejas las cuales ayudaron a la fecundación y al posterior cuajado de los frutos. Este resultado coincide con Zaccari (2004) quien indica que las abejas constituyen los agentes más eficientes en la polinización de las cucurbitáceas. Cuando las abejas visitan las flores para acopiar néctar y polen transfieren este último entre las estructuras reproductivas lo que inicia el proceso de formación de semillas o frutos.

En el tratamiento T2 (túnel con malla antiáfidos) sin presencia de abejas el número de frutos por planta es inferior al resto de los tratamientos con una media de 2.63 frutos. Esto puede deberse a la falta de agentes polinizadores ya que en los ecosistemas agrícolas los polinizadores silvestres son escasos para asegurar una adecuada polinización.

\subsection{Tamaño del fruto}

En el análisis de varianza para el tamaño de frutos en cm (Tabla 9) se encuentran diferencias altamente significativas para los tratamientos con un p-valor < 0,0001; su coeficiente de variación alcanzó un 0,72\%.

Tabla 9. Análisis de varianza para tamaño de frutos $(\mathrm{cm})$

\begin{tabular}{|c|c|c|c|c|c|}
\hline F.V. & SC & GL & $\mathbf{C M}$ & $\mathbf{F}$ & P-valor \\
\hline Repeticiones & 0,18 & 2 & 0,09 & 1,97 & 0,2541 \\
\hline Tratamientos & 1303,24 & 2 & 651,62 & 14106,05 & $<0,0001$ \\
\hline Error & 0,18 & 4 & 0,05 & & \\
\hline Total & 1303,61 & 8 & & & \\
\hline C.V. $0,72 \%$ & & & & & \\
\hline
\end{tabular}

En la prueba de Tukey al 5\% para tamaño de fruto (Tabla 10) se presentan tres rangos: en el rango "A" se encuentra el tratamiento T1 (túnel con malla entomológica) con una media de 41,70 y en el rango "C" el T2 (túnel con malla antiáfidos) con una media $13,25 \mathrm{~cm}$.

Tabla 10. Prueba de Tukey al 5\% para el tamaño de frutos

\begin{tabular}{|c|c|c|}
\hline Tratamiento & Medias & Rango \\
\hline (T1) Túnel con malla entomológica & 41,70 & $\mathrm{~A}$ \\
(T3) Campo abierto & 34,16 & $\mathrm{~B}$ \\
(T2) Túnel con malla antiáfidos & 13,25 & $\mathrm{C}$ \\
\hline
\end{tabular}

El tratamiento T1 (tuúnel con malla entomológica), con presencia de abejas (Tabla 10), presenta el mayor tamaño del fruto por planta, con una media de $41,70 \mathrm{~cm}$. Esto puede deberse a una buena polinización por la presencia de abejas en el túnel, lo que coincide con Reyes \& Cano(2005) quienes mencionan que los frutos de buena calidad de cucurbitáceas tienen muchas semillas por efecto de una buena polinización.

El tratamiento T2 (túnel con mallaantiáfidos), sin presencia de abejas, obtuvo el menor tamaño con una media de $13.25 \mathrm{~cm}$ de longitud, la cual no es muy apreciada en el mercado por el consumidor. Lo anteriormente expuesto 
concuerda con Reyes y Cano (2005) quienes indican que la malformación de frutos y tamaños pequeños, son el resultado de la polinización inadecuada (poco número de óvulos fertilizados). También coincide con CDA (2004) quien señala que los frutos de buena calidad de cucurbitáceas tienen muchas semillas.

La malformación de frutos y tamaños pequeños puede ser, algunas veces, el resultado de la polinización inadecuada (poco número de óvulos fertilizados). Hay una relación muy estrecha ente tamaño y forma de frutas y cantidad de semilla. Los insectos, especialmente las abejas, son los mejores agentes polinizadores por su facilidad de manejarlos, porque se pueden introducir al cultivo, ubicar exactamente cuando y donde se requiere. Por lo tanto, la polinización de abejas en todas las variedades de cucurbitáceas es esencial para una buena producción.

\subsection{Diámetro del fruto}

En el análisis de varianza para el diámetro del fruto en cm (Tabla 11) se encuentran diferencias altamente significativas para los tratamientos con un p-valor < 0,0001; su coeficiente de variación alcanzó un 1,41\%.

Tabla 11. Análisis de varianza para el diámetro del fruto en $(\mathrm{cm})$

\begin{tabular}{|c|c|c|c|c|c|}
\hline F.V. & SC & GL & CM & F & P-valor \\
\hline Repeticiones & 0,02 & 2 & 0,01 & 1,00 & 0,4444 \\
Tratamientos & 50,76 & 2 & 25,38 & 2376,78 & $<0,0001$ \\
Error & 0,04 & 4 & 0,01 & & \\
Total & 50,82 & 8 & & & \\
\hline C.V. $1,41 \%$ & \multicolumn{6}{l}{} \\
\hline \multicolumn{6}{|c|}{$\mathrm{p}<0,05^{*} ; \mathrm{p}<0,011^{* *} ; \mathrm{p}>0,05 \mathrm{~ns}$}
\end{tabular}

En la prueba de Tukey al 5\% para el diámetro del fruto (Tabla 12) se presentan tres rangos; en el rango "A" se encuentra el tratamiento T1 (túnel con malla entomológica) con una media de 9,70 y en el rango " $\mathrm{C}$ ” el T2 (túnel con malla antiáfidos) con una media de $4,10 \mathrm{~cm}$.

Tabla 12. Prueba de Tukey al 5\% para el diámetro de fruto en $(\mathrm{cm})$

\begin{tabular}{|l|l|l|}
\hline Tratamiento & Medias & Rango \\
\hline (T1) Túnel con malla entomológica & 9,70 & A \\
(T3) Campo abierto & 8,26 & B \\
(T2) Túnel con malla antiáfidos & 4,10 & C \\
\hline
\end{tabular}

El tratamiento T1 (Túnel con malla entomológica) con presencia de abejas (Tabla 12) obtuvo el mayor diámetro de fruto con una media de $9.7 \mathrm{~cm}$. En el tratamiento T2 (túnel con malla antiáfidos), se presenta un menor diámetro de fruto con una media de 4,1 de diámetro por planta, debido a que no existieron insectos polinizadores como las abejas que ayudaran en la fecundación de sus enormes flores. El mayor diámetro puede deberse a la mejor polinización ocasionada por las abejas lo que coincide con CDA(2004) 
quien señala que los frutos de buena calidad de cucurbitáceas tienen muchas semillas. La mal formación de frutos y tamaños pequeños puede ser, algunas veces, el resultado de la polinización inadecuada (poco número de óvulos fertilizados). Hay una relación muy estrecha ente tamaño y forma de frutas y cantidad de semilla, Los insectos, especialmente las abejas, son los mejores "Agentes Polinizadores". Entonces la polinización de abejas en todas las variedades de Cucurbitáceas es esencial para una buena producción. Las abejas son los mejores polinizadores por su facilidad de manejarlos, porque se pueden introducir al cultivo, ubicar exactamente cuando y donde se requiere.

\subsection{Peso del fruto/planta}

En el análisis de varianza para peso del fruto/planta en gramos (Tabla 13) se encuentran diferencias altamente significativas para los tratamientos con un p-valor < 0,0001; su coeficiente de variación alcanzó un 0,02\%.

Tabla 13. Análisis de varianza para el peso del fruto/planta en gramos

\begin{tabular}{|l|l|l|l|l|l|}
\hline F.V. & SC & GL & CM & F & P-valor \\
\hline Repeticiones & 0,06 & 2 & 0,03 & 0,73 & 0,5352 \\
Tratamientos & 5107642,37 & 2 & 2553821,19 & 59980142,29 & $<0,0001$ \\
Error & 0,17 & 4 & 0,04 & & \\
Total & 5107642,60 & 8 & & \\
\hline C.V. 0,02\% & $\mathrm{p}<0,05 * ; \mathrm{p}<0,01 * * ; \mathrm{p}>0,05 \mathrm{~ns}$ \\
\hline \multicolumn{5}{|r|}{}
\end{tabular}

En la prueba de Tukey al 5\% para peso del fruto/planta (Tabla 14) se presentan tres rangos; en el rango "A" se encuentra el tratamiento T1 (túnel con malla entomológica) con una media de 1994,77 y en el rango "C" el T2 (túnel con malla antiafidos) con una media de 197,53 gramos.

Tabla 14. Prueba de Tukey al 5\% para el peso del fruto/planta en gramos

\begin{tabular}{|l|r|c|}
\hline \multicolumn{1}{|c|}{ Tratamiento } & Medias & \multicolumn{1}{c|}{ Rango } \\
\hline (T1) Túnel con malla entomológica & 1994,77 & A \\
(T3) Campo abierto & 1458,48 & B \\
(T2) Túnel con malla antiáfidos & 197,53 & C \\
\hline
\end{tabular}

El mayor peso de frutos por planta lo presenta el tratamiento T1 (túnel con malla entomológica) con presencia de abejas (Tabla 14), con una media de 1994,77 gramos. El tratamiento T2 (túnel con mallaantiáfidos) sin presencia de abejas presentó el menor peso de fruto con una media de 197,53 gramos. El tratamiento T1, al ser polonizadas las flores por abejas, está asegurando una mejor calidad de fruto, como el color, forma y un buen peso. Estos resultados coinciden con Cano et al. (2002) quien manifiesta que las flores hembras deben ser polinizadas por los insectos para amarrar el fruto, de lo contrario no habrá producción. Las abejas son las más importantes polinizadores permitiendo una mayor cantidad del polen transferido al estigma, lo cual es proporcional al número de semillas y el peso del fruto. 
También coincide con Ávila (2010) quien señala que si no hay buena polinización baja el porcentaje de cuaje de frutos y sale mucha fruta malformada.

\subsection{Rendimiento en $\mathrm{kg} / \mathrm{ha}$ por tratamiento}

En el análisis de varianza para rendimiento en $\mathrm{kg} / \mathrm{ha}$ por tratamiento (Tabla 15) se encuentran diferencias altamente significativas para los tratamientos con un p-valor < 0,0001; su coeficiente de variación alcanzó un $0,02 \%$.

Tabla 15. Análisis de varianza para rendimiento en $\mathrm{kg} / \mathrm{ha}$ por tratamiento

\begin{tabular}{|l|l|l|l|l|l|}
\hline F.V. & SC & GL & CM & F & P-valor \\
\hline Repeticiones & 2,41 & 2 & 1,20 & 0,60 & 0,5918 \\
Tratamientos & 283704806,32 & 2 & 141852403,41 & 70651445,59 & $<0,0001$ \\
Error & 8,03 & 4 & 2,01 & & \\
Total & 283704817,26 & 8 & & & \\
\hline C.V. 0,02\% & $\mathrm{p}<0,05 * ; \mathrm{p}<0,01 * * ; \mathrm{p}>0,05 \mathrm{~ns}$ & \\
\hline \multicolumn{5}{|c|}{}
\end{tabular}

En la prueba de Tukey al 5\% para rendimiento en $\mathrm{kg} / \mathrm{ha}$ por tratamiento (Tabla 16) se presentan tres rangos; el rango " $\mathrm{A}$ " se encuentra el tratamiento T1 (túnel con malla entomológica) con una media de 14866,53 y en el rango "C" el T2 (túnel con malla antiáfidos) con una media de 1472,07 kh/ha.

Tabla 16. Prueba de Tukey al 5\% para el peso del fruto/planta en gramos

\begin{tabular}{|l|l|l|}
\hline Tratamiento & Medias & Rango \\
\hline (T1) Túnel con malla entomológica & 14866,53 & A \\
(T3) Campo abierto & 10869,97 & \multicolumn{1}{|c|}{ B } \\
(T2) Túnel con malla antiáfidos & 1472,07 & C \\
\hline
\end{tabular}

El tratamiento T1 (túnel con malla entomológica) con presencia de abejas obtuvo el mayor rendimiento con 14866.,53 kg/ha. (Tabla 16), mientras que en el tratamiento T2 (Túnel con malla antiáfidos) sin presencia de abejas se obtuvo el menor rendimiento con $1472.1 \mathrm{~kg} / \mathrm{ha}$. El alto rendimiento obtenido en el tratamiento $\mathrm{T} 1$ puede deberse a la presencia de (Apis mellifera) como agentes polinizadores, los cuales incrementaron el rendimiento y mejoró la calidad de fruto. Esto coincide con ACTAD (2008) quien señala que se pueden obtener rendimientos que oscilan entre 12 y 14 t.ha-1 con la polinización con las abejas. Se coincide también con Garibaldi et al. (2012), quien manifiesta que las cucurbitáceas (zapallos, sandías, melones, entre otros) también constituyen un ejemplo de alta dependencia de polinizadores, particularmente de abejas grandes que actúan de manera efectiva en sus enormes flores. Sin embargo, la mayoría de los cultivos depende parcialmente de polinizadores. La desaparición de estos produciría una reducción limitada, aunque importante, de la producción, como por 
ejemplo en Argentina cuyo rendimiento disminuiría más de $40 \%$ en ausencia de polinizadores.

\section{Conclusiones}

Los mejores promedios en: porcentaje de flores fecundadas con un $84 \%$, número de frutos por planta con 6.7 frutos cosechados, en el tamaño de fruto con $41.7 \mathrm{~cm}$ de longitud y un diámetro de $9,7 \mathrm{~cm}$, peso del fruto de 1994.8 gramos que equivale a $1.9 \mathrm{~kg}$ por planta alcanzando un rendimiento de $14866.5 \mathrm{~kg} /$ ha que equivale a $14.9 \mathrm{tn} / \mathrm{ha}$ se obtuvieron en el Tratamiento T1 (túnel con malla entomológica).

El mejor rendimiento se obtuvo con el tratamiento T1 (túnel con malla entomológica) con presencia de abejas con $14866 ., 53 \mathrm{~kg} / \mathrm{ha}$ y el menor rendimiento con $1472.1 \mathrm{~kg} / \mathrm{ha}$ se obtuvo en el tratamiento T2 (túnel con malla antiáfidos) sin presencia de abejas.

\section{Agradecimientos}

Al equipo científico de la Escuela Superior Politécnica de Chimborazo, por su valiosa contribución científica y técnica.

\section{References:}

1. ACTAD (2008). Influencia de las abejas en el rendimiento de calabacín. Instituto técnico de cultivo de calabaza. Publicaciones Azucareras. Segunda Edición. La Habana. Cuba. 15p.

2. Arizmendi (2009). http://oaji.net/articles/2016/2674-1455221973.pdf

3. Ávila, R. (2010). Entrenamiento y desarrollo de agricultores. Consultado 27/12/2018. Obtenido de http://repositorio.uaaan.mx:8080/xmlui/bitstream/handle

4. Canon et al. (2002). Producción y calidad del fruto melón bajo diferentes periodos de polinización. Asociación de veterinarios especialistas en abejas. A.C, Vol 35 (4) 80-85p.

5. CDA (2004). Las abejas en relación a la polinización en las cucurbitáceas. Oficina de agricultura y recursos naturales de la agencia nacional de los estados unidos para el desarrollo del estado internacional. Boletín técnico $\mathrm{N}^{\circ}$ 53. Estados Unidos 1-3p

6. FAO (2014). Principios y avances sobre polinización como servicio ambiental para la agricultura.

7. Garibaldi et al. (2012). Los polinizadores en la agricultura. Vol 21(126). 35-43p. Consultado 14/08/2018. Obtenido https://www.researchgate.net

8. Garibaldi et al. (2013). Wild Pollinators Enhance Fruit Set of Crops Regardless of Honey Bee Abundance. Science. 2013;339(6127):16081611. Doi: $10.1126 /$ science. 1230200 
9. Hamilton et al. (2010). Quantifying Uncertainty in Estimation of Tropical Arthropod Species Richness. $\mathrm{T}$ Am Naturalist. 2010;176(1):90-95.

10. Kevan, P. (2003). Forest application of the insecticide Fenitrothion and its effect on wild bee pollinators (Hymenoptera: Apoidea) of lowbush blueberries (Vaccinium spp.) in Southern New Brunswick, Canada. Biol. Conserv. 7, 301-309

11. Martínez (2009). Cucurbitaceas. Consultado 22/08/2018. Obtenido de Botanical-online: http://www. botanical online.com/familiacucubitaceascastella.

12. Moacho, F. (2011). Cultivo de cucurbitáceas. Obtenido de http://felixmaocho.wordpress.com/2011 /08/21/huerto-familiarcultivo-de-la-calabaza/

13. Nepi, M. \& Pacini, E. (1993). Pollination, pollen viability and pistil receptivity in Cucurbita pepo. Ann of Bot 72: 527-536

14. Noriega, E. (2003). Manual de fertilizantes para la horticultura (Segunda ed.). Mexico.

15. Reyes \& Cano (2005). Manual de Polinización Apícola. La polinización de los cultivos por abejas. Universidad Autónoma Agraria Antonio Narro, Saltillo, Coah., México. 2005, 58 p.

16. Rylski \& Alon (1990). Cucurbitáceas. Consultado 02/01/2019. Obtenido www.repositorio.ual.es/bitstream/handle/10835/3233/Trabajo577.pdf

17. Zaccari, F. (2004). Cucurbitácea. Obtenido de http://www.fagro.edu.uy/ horticultura /CURSO $\% 20$ HORTICULTURA/CUCURBITACEAS/Cucurbitaceas\%20Introduc cion\%20 Fisiologia.pdf 